Case Report

\title{
Left Atrial Myxoma: A Rare Nonatherosclerotic Cause of Acute Myocardial Infarction
}

\author{
Rey Francisco Arcenas ${ }^{1}$ and Mir Ishtiaque $\mathrm{Ali}^{2}$ \\ ${ }^{1}$ Third Year Resident Department of Internal Medicine, Indiana University Health Ball Memorial Hospital, Muncie, IN 47303, USA \\ ${ }^{2}$ Indiana University Health Ball Memorial Hospital, Muncie, IN 47303, USA
}

Correspondence should be addressed to Rey Francisco Arcenas; reyfarcenas@yahoo.com

Received 30 April 2013; Accepted 5 June 2013

Academic Editors: R. Akdemir, M. Barros, K. N. Blackett, M.-H. Jim, T. Kasai, and G. Minardi

Copyright (c) 2013 R. F. Arcenas and M. I. Ali. This is an open access article distributed under the Creative Commons Attribution License, which permits unrestricted use, distribution, and reproduction in any medium, provided the original work is properly cited.

Myocardial infarction from coronary artery embolism is a very rare but potentially lethal sequel of left atrial myxoma. Most atrial myxomas causing myocardial infarction are diagnosed retrospectively after a $2 \mathrm{D}$ echocardiogram is performed for assessment of myocardial function after a myocardial infarction. We present a relatively healthy 53-year-old male with anterolateral wall myocardial infarction and $100 \%$ occlusion at the proximal part of the obtuse marginal branch of the circumflex coronary artery that was subsequently reperfused. A 2D echocardiogram performed two days later revealed a left atrial mass, which was successfully resected and proven to be a myxoma. No recurrence of the tumor was seen on follow-up after four months. An automatic implantable cardioverter defibrillator was placed for residual ischemic cardiomyopathy with clinical improvement.

\section{Introduction}

Primary cardiac neoplasms are rare, with an incidence of less than $0.2 \%$ in unselected patients at autopsy [1]. The majority of these tumors are benign myxomas located in the left atrium $(80 \%)$ [2]. Rare coronary artery embolization from a cardiac myxoma usually involves either the right coronary artery (RCA) or the left anterior descending (LAD) coronary artery [3]. We report a case of a patient with $100 \%$ occlusion of the left circumflex (LCX) artery from a left atrial myxoma presenting as anterolateral wall myocardial infarction.

\section{Case Report}

A 53-year-old male with no significant past medical history presented with severe chest pain after exertion, associated with diaphoresis, dyspnea, cough, and three episodes of syncope. He denied smoking, use of alcohol, and illicit substances. He has no family history of coronary artery disease. On admission, he was in mild distress with blood pressure of $115 / 70 \mathrm{mmHg}$, heart rate of 100 beats per minute, respiratory rate of 20 breaths per minute, and temperature of $97.2^{\circ} \mathrm{F}$. Body mass index was $30 \mathrm{~kg} / \mathrm{m}^{2}$. There was no jugular venous distention. He had bibasilar rales on lung exam. Cardiac examination revealed a normal heart rate, regular rhythm with no clicks, gallops, or murmur. The rest of the physical examination was normal.

The 12-lead electrocardiogram showed ST-segment elevation in the anterolateral leads with reciprocal changes in the inferior leads. Chest radiograph was normal. Initial set of cardiac enzymes were normal as he presented within 30 minutes from onset of chest pain. Cardiac catheterization revealed no evidence of coronary artery disease in the RCA, left main coronary artery, nor the LAD artery. However, the proximal obtuse marginal branch of the left circumflex (LCX) artery was noted to have a complete occlusion (Figure 1).

During percutaneous intervention, the occluded segment was opened with difficulty due to the significant amount of thrombus, employing multiple balloons, and aspiration with an Export catheter. After placement of two bare-metal stents, TIMI grade 3 (normal) flow with limited myocardial blush was observed (Figure 2). Left ventricular ejection fraction (LVEF) was approximately $30 \%$ measured by left ventriculography.

A transthoracic 2D echocardiogram performed two days after the intervention revealed a very large irregular mobile 




FIGURE 1: Coronary angiogram showing the obtuse marginal branch of the left circumflex artery with $100 \%$ occlusion (white arrow).

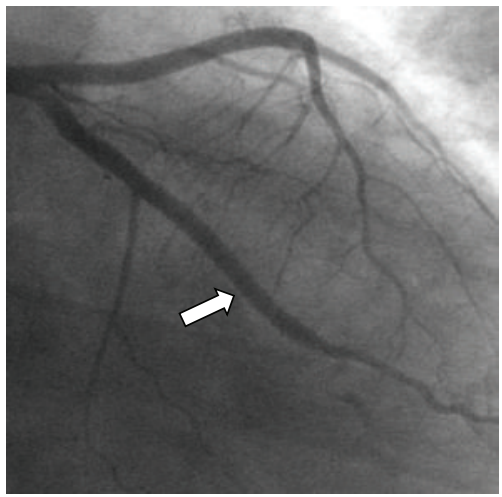

FIGURE 2: After a successful percutaneous coronary intervention, the occluded segment of the proximal portion of the obtuse marginal artery was fully reperfused (white arrow).

mass attached to the superior atrial septum freely prolapsing to the left ventricle (Figures 3 and 4). Severe hypokinesia of the apical, lateral, and midanterolateral walls were noted. LVEF is approximately $15-20 \%$.

In the succeeding days, the patient developed various complications including cardiogenic shock that needed intraaortic balloon pump placement, pneumonia, and an episode of ventricular tachycardia. These led to a delay of the definitive surgical intervention. Repeat coronary angiography showed patent stents with TIMI grade flow of 2 to 3 and limited myocardial blush. He subsequently underwent successful resection of the left atrial mass twelve days after his presentation. Histopathology confirmed the mass as an atrial myxoma.

Four months later, he was readmitted due to ventricular tachycardia; symptoms included mild dyspnea on ordinary activity. Repeat $2 \mathrm{D}$ echocardiogram revealed no recurrence of the myxoma. LVEF remained at $20 \%$ and still with significant anterior wall hypokinesia. An automatic implantable cardioverter defibrillator was subsequently placed. The patient has remained well since that time.



FIGURE 3: Two-dimensional echocardiogram demonstrates an irregular mass in the left atrium during the end-systolic phase of the cardiac cycle (white arrow).

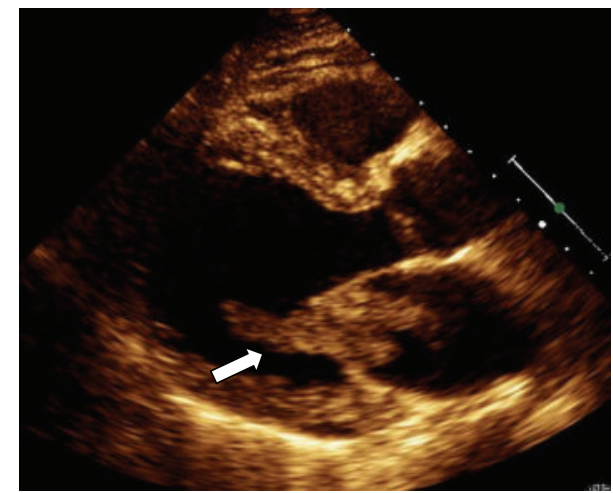

FIGURE 4: Two-dimensional echocardiogram demonstrates the irregular mass prolapsing to the left ventricle during the middiastolic phase of the cardiac cycle (white arrow).

\section{Discussion}

From our review of the literature, we found two reported cases of combined left circumflex (LCX) artery and left anterior descending (LAD) artery embolism from an atrial myxoma causing myocardial infarction (MI) $[4,5]$, one case affecting both LCX and diagonal branches of the LAD artery [6] and only three cases affecting the LCX artery alone [7-9].

Embolization from an atrial myxoma is frequently seen clinically, occurring in $32 \%$ of cases as the presenting symptom in one series [10]. However, the incidence of coronary embolization from atrial myxoma is only $0.06 \%$ [11]. Possible explanations for this very low incidence include the perpendicular disposition of the coronary ostia in relation to the aortic blood flow and the protective effect of the aortic valve leaflets during systole [12].

Coronary artery embolism from a myxoma with resultant myocardial infarction is exceedingly rare [13-15]. Its true incidence is probably underestimated from the lack of systematic echocardiographic evaluation of patients after MI. It occurs over a wide age range, from 9 years [16] to 68 years of age [5, 17-19]. 
The lesion in the obtuse marginal branch in our patient cannot account for the electrocardiographic changes of anterolateral MI. The most likely explanation is that the emboli may have lodged temporarily in the left main coronary artery obstructing both the LAD and LCX arteries before it migrated down. It is also possible that he had a concomitant LAD embolization that broke off into smaller fragments or spontaneously lysed, accounting for a patent LAD artery on angiography [13, 20-22].

Several other characteristics of a myxoma are described in the literature. It is well documented that tumor emboli have malignant potential $[6,23-25]$, with the ability to persist [9], invade, and even grow inside the coronary artery. Conversely, there are cases of left atrial myxoma presenting as acute MI but with normal coronary arteries. Braun et al. [26] noted that a normal coronary artery despite a clinical diagnosis of acute embolic MI is still possible due to the myxomatous histology of the tumor, allowing it to embolize and resolve spontaneously. This makes the timing of symptom onset and the actual presentation critical for accurate diagnosis. Isobe et al. [27] cited the ability of myxomas to secrete interleukins 6 and 8 that may cause systemic manifestations like fever and cachexia. Our patient did not have any systemic manifestation prior to presentation.

The diagnosis of atrial myxoma causing MI is seldom established prior to cardiac catheterization, with rare exceptions [10]. Most cases have unrevealing cardiac examination findings which would prompt an echocardiogram. In other cases, a loud S1, a short presystolic murmur, and an apical middiastolic sound prompted echocardiographic evaluation $[11,28]$. The diastolic sound is produced as the tumor strikes the ventricular wall and is characteristically intermittent [29]. The myxoma may obstruct the mitral valve and cause syncope, as in this patient. Rarely, neovascularization of the tumor mass may give a clue to its presence during cardiac catheterization [13].

Most atrial myxomas associated with myocardial infarction are discovered incidentally by $2 \mathrm{D}$ echocardiogram done to assess myocardial function after an event. Early echocardiography in all patients with a first episode of pulmonary edema [30] or acute coronary syndrome, especially in a relatively young patient with few risk factors, will expedite the diagnoses in most if not all cases. It is important to diagnose this condition because surgical intervention is the only means to improve the prognosis [18].

Additional concerns with an undiagnosed left atrial myxoma causing MI are the risk of extensive hemorrhagic MI with tamponade [10] and tumor fragmentation with further systemic embolization [28] when thrombolysis is performed.

Almost all cases previously reported had relatively good outcome after surgical intervention. However, some cases have complicated courses before the surgery, such as what happened to our patient. Tomita et al. [10] reported a case of a patient who died from multiorgan failure despite the use of intra-aortic balloon pump, due to extensive hemorrhagic myocardial infarction from a myxoma. Surgical resection is usually sufficient and curative with $0-3 \%$ operative mortality rate [1]. Resection of the tumor with percutaneous coronary intervention or coronary artery bypass surgery is needed when there is subtotal or total occlusion of a coronary artery [31]. And because there is a $2-5 \%$ recurrence rate for myxomas, [1] follow-up with $2 \mathrm{D}$ echocardiography is necessary.

\section{Conclusion}

Myocardial infarction from embolization by an atrial myxoma, although very rare, highlights the need to consider myxoma as one of the rare nonatherosclerotic causes of MI. This condition is lethal if not diagnosed and treated in a timely manner. $2 \mathrm{D}$ echocardiography after a myocardial infarction is the most efficient way to diagnose atrial myxoma even among those without symptoms. Surgical resection of the myxoma with percutaneous coronary intervention or coronary artery bypass grafting for patients with significant coronary occlusion is essential to improve the prognosis of the patient.

\section{References}

[1] K. Reynen, "Medical progress: cardiac myxomas," New England Journal of Medicine, vol. 333, no. 24, pp. 1610-1617, 1995.

[2] K. Verkkala, M. Kupari, T. Maamies et al., "Primary cardiac tumours-operative treatment of 20 patients," Thoracic and Cardiovascular Surgeon, vol. 37, no. 6, pp. 361-364, 1989.

[3] M. Namura, H. Kanaya, H. Sanada, T. Tsuchiya, and T. Ohka, "Left atrial myxoma presenting as acute myocardial infarction," Japanese Journal of Medicine, vol. 29, no. 5, pp. 537-541, 1990.

[4] A. H. M. Balk, S. S. Wagenaar, and A. V. G. Bruschke, "Bilateral cardiac myxomas and peripheral myxomas in a patient with recent myocardial infarction," American Journal of Cardiology, vol. 44, no. 4, pp. 767-770, 1979.

[5] N. A. Hoad and J. S. Foulds, "Fortuitous discovery of a left atrial myxoma following acute myocardial infarction," Journal of the Royal Army Medical Corps, vol. 133, no. 1, pp. 13-15, 1987.

[6] N. Konagai, M. Cho, K. Nakamura, and H. Shigematsu, "Left atrial myxoma as a cause of acute myocardial infarction," Texas Heart Institute Journal, vol. 37, no. 1, pp. 125-126, 2010.

[7] M. N. Attar, R. K. Moore, and S. Khan, "Left atrial myxoma presenting with ventricular fibrillation," Journal of Cardiovascular Medicine, vol. 9, no. 3, pp. 282-284, 2008.

[8] H. M. van Gelder, J. P. Jacobs, and J. McCormack, "Acute myocardial infarction in a 15 -year old secondary to myxomatous embolisation," Cardiology in the Young, vol. 14, no. 6, pp. 658-660, 2004.

[9] G. Milicevic, Z. Gavranovic, H. Cupic et al., "Unremitting embolus from cardiac myxoma at circumflex artery trifurcation," International Journal of Cardiology, vol. 126, no. 3, pp. 424-426, 2008.

[10] Y. Tomita, T. Endo, T. Takano, H. Hayakawa, T. TamuraK., and Y. Sugisaki, "Extensive hemorrhagic myocardial infarction associated with left atrial myxoma," Cardiology, vol. 81, no. 6, pp. 384-388, 1992.

[11] K. L. Lehrman, G. B. Prozan, and D. Ullyot, "Atrial myxoma presenting as acute myocardial infarction," American Heart Journal, vol. 110, no. 6, pp. 1293-1295, 1985.

[12] A. Panos, A. Kalangos, and J. Sztajzel, "Left atrial myxoma presenting with myocardial infarction: case report and review 
of the literature," International Journal of Cardiology, vol. 62, no. 1, pp. 73-75, 1997.

[13] S. Rath, Y. Har-Zahav, and A. Battler, "Coronary arterial embolus from left atrial myxoma," American Journal of Cardiology, vol. 54, no. 10, pp. 1392-1393, 1984.

[14] J. Silverman, J. S. Olwin, and J. S. Graettinger, "Cardiac myxomas with systemic embolization. Review of the literature," Circulation, vol. 26, pp. 99-103, 1962.

[15] D. L. Price, J. L. Harris, P. F. New, and R. C. Cantu, "Cardiac myxoma. A clinicopathologic and angiographic study," Archives of Neurology, vol. 23, no. 6, pp. 558-567, 1970.

[16] S. Harikrishnan, S. R. KrishnaManohar, R. Krishna Kumar, and J. M. Tharakan, "Left atrial myxoma presenting as acute myocardial infarction in a child," Cardiology, vol. 99, no. 1, pp. 55-56, 2003.

[17] K. Durgut, N. Görmüs, M. Ozulku, U. Özergin, and C. Özpinar, "Clinical features and surgical treatment of cardiac myxoma: report of 18 cases," Asian Cardiovascular and Thoracic Annals, vol. 10, no. 2, pp. 111-114, 2002.

[18] C. Tóth and M. Lengyel, "Images in cardiology: acute myocardial infarction as first manifestation of left atrial myxoma," Acta Cardiologica, vol. 57, no. 5, pp. 365-366, 2002.

[19] K. Uemura, T. Hiramatsu, T. Kuzi, H. Satoh, and T. Tomino, "A case of left atrial myxoma with acute myocardial infarctionemergency coronary artery bypass grafting and removal of left atrial myxoma," Journal of the Japanese Association for Thoracic Surgery, vol. 41, no. 8, pp. 1386-1389, 1993.

[20] H. Hashimoto, H. Takahashi, Y. Fujiwara, T. Joh, and T. Tomino, "Acute myocardial infarction due to coronary embolization from left atrial myxoma," Japanese Circulation Journal, vol. 57, no. 10, pp. 1016-1020, 1993.

[21] Y. Soejima, A. Niwa, M. Tanaka et al., "A left atrial myxoma complicated with acute myocardial infarction," Internal Medicine, vol. 36, no. 1, pp. 31-34, 1997.

[22] M.-H. Namazee, H.-R. Rohani-Sarvestani, and A.-R. Serati, "The early presentation of atrial myxoma with acute myocardial infarction," Archives of Iranian Medicine, vol. 11, no. 1, pp. 98102, 2008.

[23] G. Budzilovich, S. Aleksic, and A. Greco, "Malignant cardiac myxoma with cerebral metastases," Surgical Neurology, vol. 11, no. 6, pp. 461-469, 1979.

[24] M. Kelly and A. G. Bhagwat, "Ultrastructural features of a recurrent endothelial myxoma of the left atrium," Archives of pathology, vol. 93, no. 3, pp. 219-226, 1972.

[25] D. Heath and J. Mackinnon, "Pulmonary hypertension due to myxoma of the right atrium: with special reference to the behavior of emboli of myxoma in the lung," American Heart Journal, vol. 68, no. 2, pp. 227-235, 1964.

[26] S. Braun, H. Schrötter, K. Reynen, C. Schwencke, and R. H. Strasser, "Myocardial infarction as complication of left atrial myxoma," International Journal of Cardiology, vol. 101, no. 1, pp. 115-121, 2005.

[27] N. Isobe, T. Kanda, H. Sakamoto, Y. Morishita, T. Suzuki, and I. Kobayashi, "Myocardial infarction in myxoma patients with normal coronary arteries: case reports," Angiology, vol. 47, no. 8, pp. 819-824, 1996.

[28] V. M. Abascal, J. Kasznica, G. Aldea, and R. Davidoff, "Left atrial myxoma and acute myocardial infarction: a dangerous duo in the thrombolytic agent era," Chest, vol. 109, no. 4, pp. 1106-1108, 1996.
[29] R. S. Zitnik and M. R. Giuliani, "Clinical recognition of atrial myxoma," American Heart Journal, vol. 80, no. 5, pp. 689-700, 1970.

[30] I. C. Gawthrope and J. A. Davidson, "A mysterious case of chest pain," Emergency Medicine Australasia, vol. 17, pp. 170-172, 2005.

[31] M. Demir, O. Akpinar, and E. Acarturk, "Atrial myxoma: an unusual cause of myocardial infarction," Texas Heart Institute Journal, vol. 32, no. 3, pp. 445-447, 2005. 


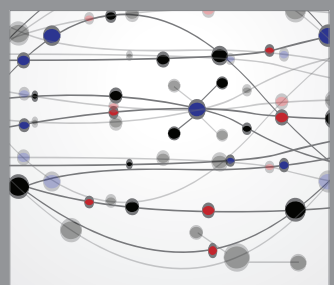

The Scientific World Journal
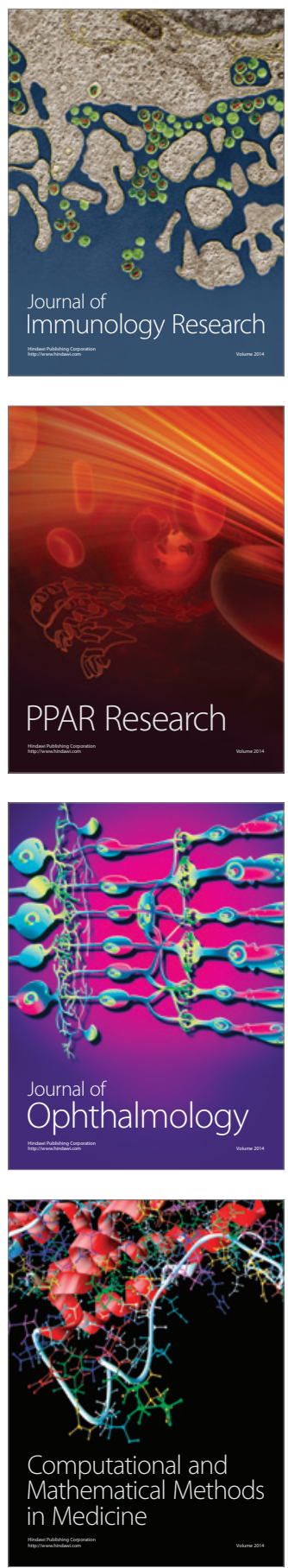



Gastroenterology

Research and Practice
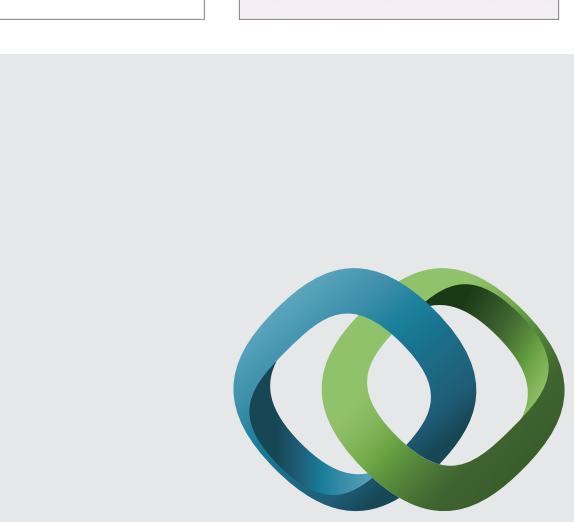

\section{Hindawi}

Submit your manuscripts at

http://www.hindawi.com
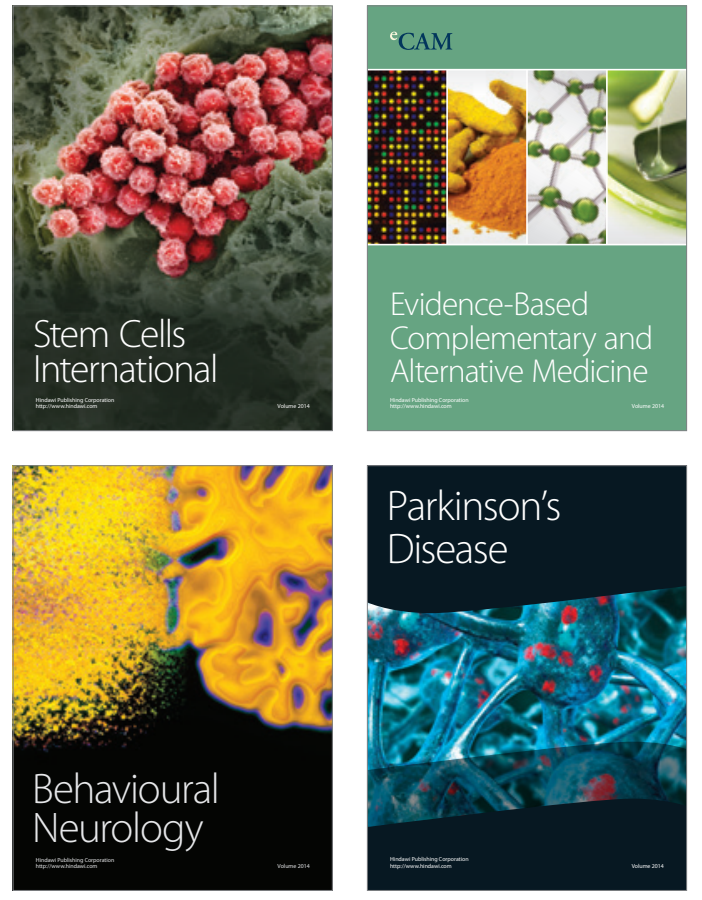
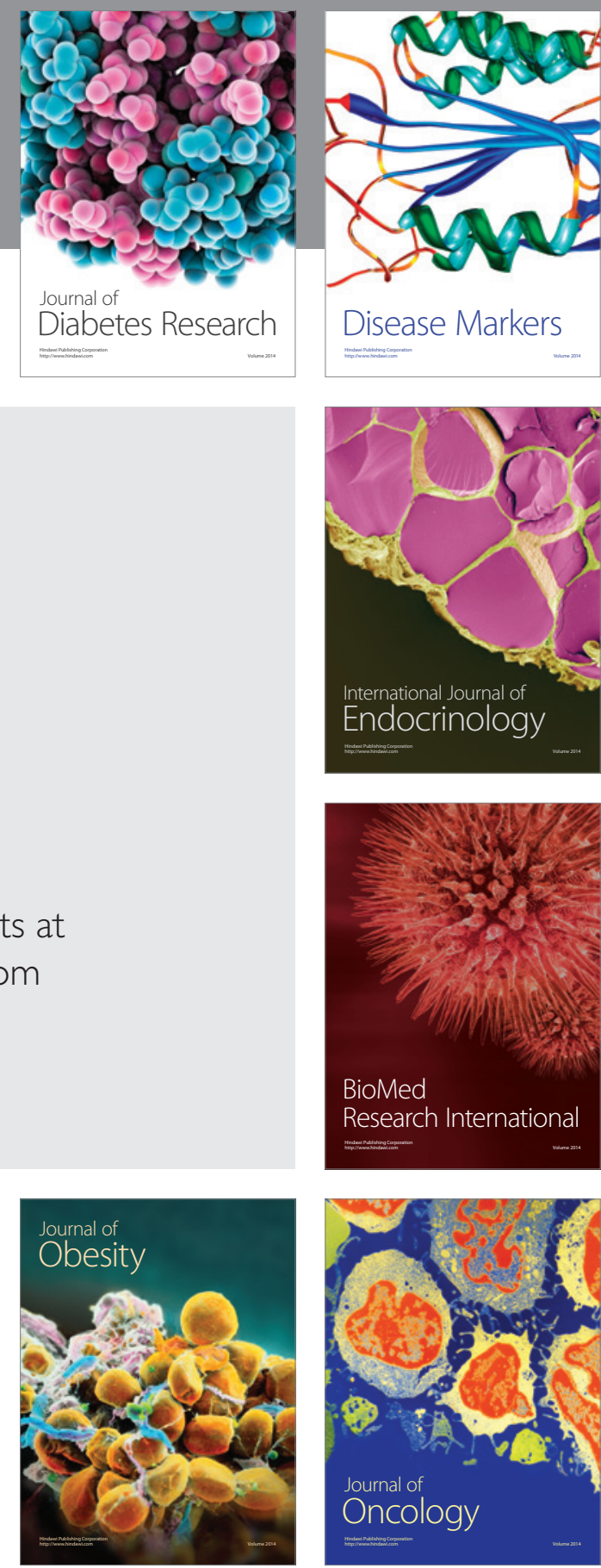

Disease Markers
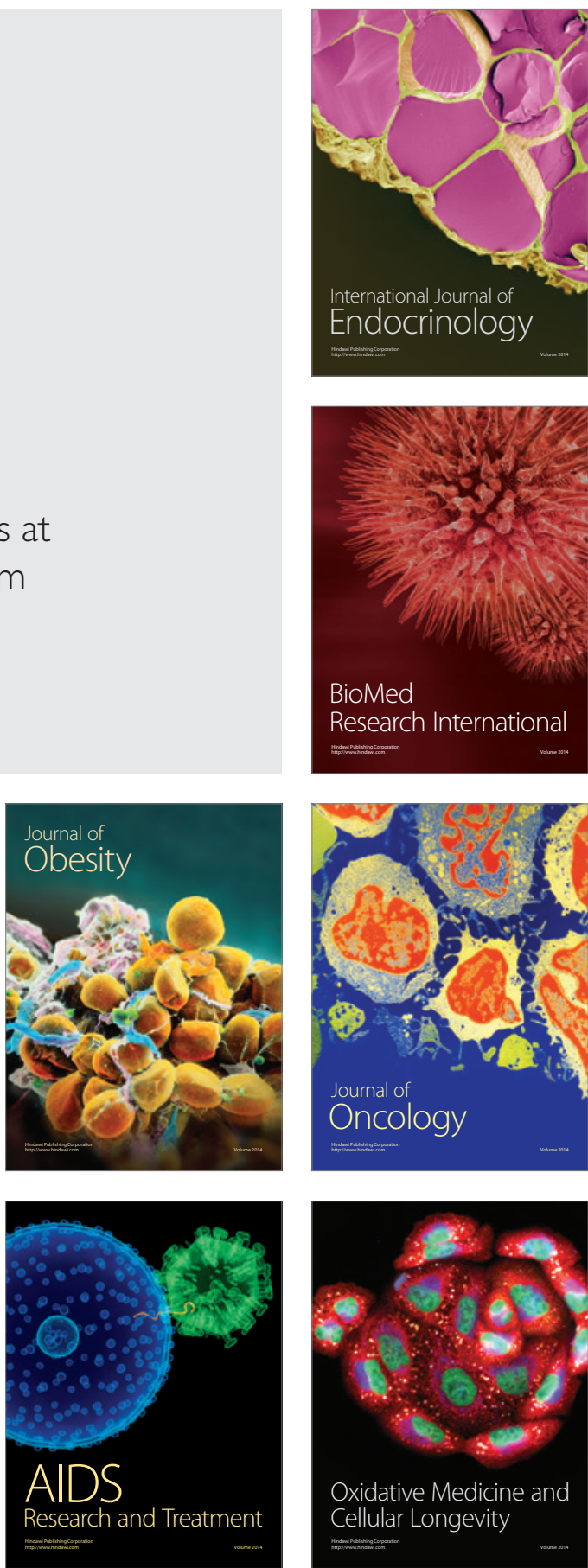